\title{
General Concepts of a Manufacturing System Engineering Workbench as a Tool for the Re-engineering of Manufacturing Systems
}

\section{Garetti $M$.}

professor of Industrial Technology

Dipartimento di Economia e Produzione, Politecnico di Milano

piazza Leonardo Da Vinci 32, 20133 Milano, Italy

phone: 39223994760 fax: 39223994805

Bartolotta A.

research fellow of the National Research Council (CNR)

Dipartimento di Economia e Produzione, Politecnico di Milano

piazza Leonardo Da Vinci 32, 20133 Milano, Italy

phone: 39223994815 fax: 39223994805

\begin{abstract}
This paper highlights the main aspects of a research program developed by the Dipartimento di Economia e Produzione of the Politecnico di Milano within the framework of a national research project on the Design and Management of Advanced Production Systems. The study aims at defining the general architecture of a workbench for the design of industrial production systems, identifying appropriate problem-solving tools and developing a prototype-version of such a system. The approach followed by the authors is based on a general descriptive method for generic production system definition, drawn from Zeigler's system entity structure. Based on this general concept, a two-stage design approach is proposed for the design and redesign activities of manufacturing systems. In the authors' opinion this kind of approach could remarkably improve the efficiency of manufacturing systems design and re-engineering tasks, which are major contributors to competitive success in modern industries worldwide.
\end{abstract}

\section{Keywords}

Object-oriented modelling of manufacturing systems, Manufacturing systems modelling, Computer-Aided Engineering, Design of manufacturing systems 


\section{INTRODUCTION}

In the past decade, companies have been forced to cope with many deep changes, most of which were caused by the increasing complexity and instability of processes on the one hand and by stronger market turbulences on the other.

Increased process complexity is linked to the broadening of the technological foundations of products and processes, to the general widening of product ranges and to an increased demand for customization.

The increase in market turbulence has placed strict constraints on the response time of the industrial system and has determined the success of time-based competitive strategies.

These deep changes have forced industries to deploy new manufacturing strategies to adjust to external pressures: hence the fast and dramatic change in the organizational and managerial criteria applied to company and manufacturing strategy. In many cases, industrial engineers have been forced to reengineer corporate manufacturing facilities in order to guarantee their long-term survival. The success of a manufacturing company is more and more closely linked to the speed and efficiency with which it can incorporate new technologies. Since past experiences have shown that information technology is one of the most effective success drivers, when re-designing processes and organizational schemes must take place (Davenport and Short, 1990), the concept of a software system capable of supporting production engineers in the design, analysis and preliminary assessment of the performance of manufacturing systems has started developing. Hereafter this system will be called a Manufacturing System Engineering Workbench, or briefly MSEW.

Such a tool appears to be quite useful for manufacturing companies which need to frequently re-engineer plants to preserve their competitiveness: in these companies production engineers must adjust to external changes frequently, or rather their adjustment cycle must be shorter than the external one (Wang et al., 1993).

The process currently used to design or redesign manufacturing systems has been poorly formalized so far. In fact available information tools are highly specialized and difficult to use; moreover they do not allow the sharing of information; thus their use is quite limited. However, considering the costs and resources involved in these activities, it is quite evident that the improvement of the re-design process would be extremely profitable. Like product designers, who have sophisticated and integrated CAD/CAM/CAE tools available, production engineers need advanced computing tools to solve their problems and manage the huge amount of data associated to the design of a manufacturing system more easily.

Therefore, besides automating routine tasks and providing critical technical data to support the necessary decision-making processes, this design environment should allow the use of integrated information tools for the computer-aided design of manufacturing systems (McLean, 1993).

Such a tool could be used to manage the continuous improvement of a manufacturing system, to store information about production resources, to improve production capacity, to design new manufacturing systems etc. In the case of a large manufacturing system, the 
structured knowledge which would be available in the system database might enable several designers to work simultaneously and to draw up detailed plans and operating models for the whole company in a few days. Many options could rapidly be assessed and implemented, with a significant improvement over current manual methods which may require weeks or months of intensive design activity.

Such a software system is not available yet, but the current level of hardware and software tools will make its implementation viable in a not-distant future.

\section{DESIGNING A MSEW}

The project of a MSEW first requires the development of a structured knowledge in the domain of manufacturing systems, i.e. a semantically structured definition of the classes of manufacturing systems to be described. Furthermore the need to integrate all the tools to be included in the workbench makes it necessary to share access to common data, i.e. a centralized information system that can be understood and accessed by all modules and tools in the system must be developed. To this purpose a general descriptive method which can be easily loaded into a computer will be suggested for the definition of a generic manufacturing system.

\subsection{Object-oriented modelling of manufacturing systems}

A manufacturing system can be divided into three different aspects:

- the structural aspect, providing a static view of the manufacturing process, is defined by the units making up the system and their relationships (lay-out schemes are a typical method used to highlight the structural aspect of a manufacturing system);

- the technological aspect, describing the conversion phases taking place within the manufacturing system, is based on a functional (i.e. dynamic) analysis of the system itself, detecting parts flowing through the system (this aspect is generally illustrated through the use of technological diagrams);

- the management aspect, describing the operating steps of the manufacturing system, is provided by the set of functions making up the actual manufacturing process (typically including planning, scheduling and control activities).

Each aspect will only capture some features of a manufacturing system, but the whole system would be exhaustively described by putting the three aspects together.

When following the principles of object-oriented programming, each aspect (i.e. the structural, technological and management aspect) of a generic manufacturing system can be described as a set of objects.

More specifically, three different object classes can be defined (see Figure 1):

- plant components

- technological methods

- management methods 


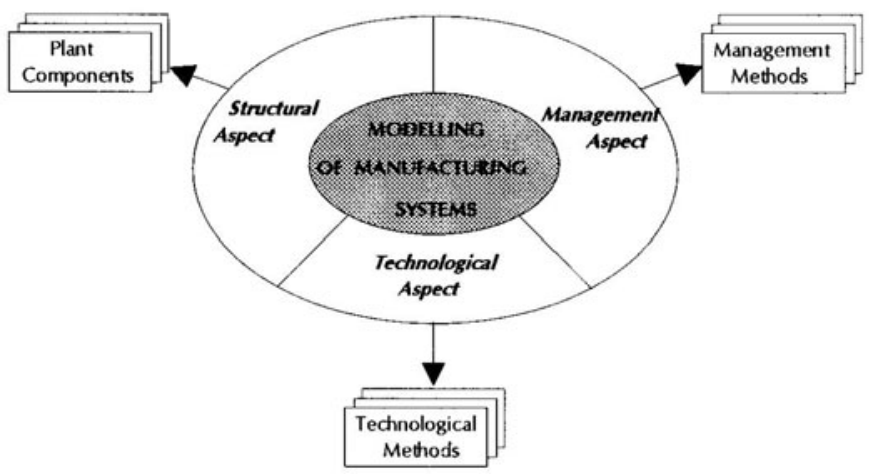

Figure 1 Modelling of a manufacturing system.

Obviously, the identification of components is more intuitive when the structural aspect is examined, since objects defining structural aspects can be quite easily matched to the physical entities making up a manufacturing system. A bigger abstraction effort is required when applying this approach to the other two aspects of the manufacturing system, i.e. the technological and the management aspects, since no matching is possible between objects and physical entities.

The convenience of such an object-oriented modelling system is quite evident: the resulting knowledge is flexible and can be easily changed, objects (plant components, technological and management methods) can be combined freely to create many different models, changes can be restricted to some objects without any need to reconfigure the whole model, etc.

Some modelling detail over this three main aspects is discussed in the following.

\section{Structural aspect}

The analysis of the structural aspect aims at detecting Plant Components and their relationships. Plant components can be divided into:

- permanent objects

- temporary objects.

Permanent objects are resources (i.e. process-shaping objects) always available within the manufacturing system, i.e. components which can be found throughout the whole process (typically machine tools, magazines and handling equipment).

Permanent objects can be divided into:

- stocking agents, that are system resources used to store of temporary objects awaiting to perform certain tasks;

- processing agents, that are system resources (operating stations) dealing with the physical processing of parts or assemblies; 
- handling agents, that are system resources used to move parts from an operating station to the next one, linking operating stations to one another, thus generating the part flow.

Temporary objects are entities (process-undergoing objects) which flow through the manufacturing system (typically parts, assemblies and pallets).

\section{Technological aspect}

Through the definition of objects called Technological Methods, the technological aspect of a manufacturing system highlights the dynamic processing behaviour of the system. While the structural aspect focuses on the permanent objects making up the system, the technological aspect focuses on temporary objects, describing the flow of parts, assemblies and pallets inside the system. The following object types are only a few examples within the technological aspect:

- technological cycle of each part type;

- cycle time required for each part type;

- setup time required before a new part type is processed;

- number of scraps for each part type at each inspection station;

- technological lot size, defined as the number of parts which can be processed in parallel by a processing agent.

\section{Management aspect}

Through the definition of objects called Management Methods, the management aspect describes the operating behaviour of the manufacturing process, i.e. the control strategies and management logics governing the interaction between permanent and temporary objects within the manufacturing system.

Generally speaking, information about the management aspect of a manufacturing system fall into the following object groups:

- mechanisms regulating the feeding of parts to the system;

- part routing, if alternative technological routes exist for a part type;

- breakdown of permanent objects;

- dispatching rules (to determine priority levels among parts waiting for a resource);

- loading rules (to determine the feeding sequence of parts to the system);

- management rules of scrap.

\subsection{Formal representation of manufacturing systems}

Based on the concepts put forth by Zeigler et al. $(1987,1989)$ the semantics developed in the previous chapter for a generic manufacturing system can be formally integrated into a representation diagram which can be easily implemented on a computer, called System Entity Structure (SES).

The System Entity Structure is a knowledge-representing scheme combining decomposition and taxonomy relationships: decomposition means explaining how an object can be broken 
down into its components; taxonomy means representing all the possible variants of an object (called specializations).

A scheme like the System Entity Structure proves very useful in describing large-scale systems, which are extremely difficult to define using conventional, highly-detailed analysis methods. Manufacturing systems definitely fall into this category. Zeigler suggests to break these systems down into several levels, which allows to see the same system from different viewpoints. Such operations are applied on different levels, thereby making it possible to understand how a system can be broken down into its component objects and how such objects can be further broken down into their components.

From a graphical viewpoint, the SES diagram appears as a tree-shaped structure, with decomposition relationships being represented as single lines and toxonomy relationships as double lines. As the example of Figure 2 describes the root of the mfg systems SES, it only shows decomposition relationships i.e. objects broken into components, this is why taxonomy relationships are present only in deeper SES levels.

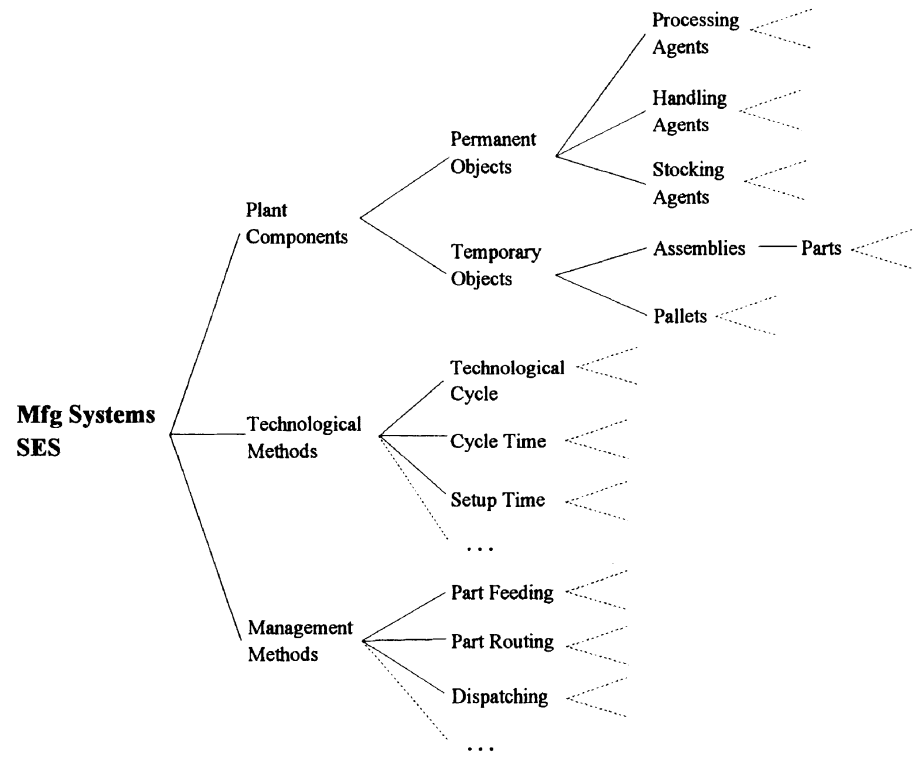

Figure 2 Root of the System Entity Structure of a generic manufacturing system.

The role played by a well-structured knowledge-representing scheme like SES in the MSEW project should not be underestimated. The possibility to store the features of the countless physical and non-physical elements making up a manufacturing system into a single data format and to manage this information in a computer-aided way are an essential prerequisite for the development of a MSEW. 


\subsection{General architecture of a MSEW}

Defining the general architecture of a Manufacturing System Engineering Workbench means developing an environment of "organized data" (including input, half-processed and output data) and operating tools which can be used by designers to process both previously stored information and new data they have entered themselves, for the computer-aided generation of a manufacturing system model.

The general architecture of the computer-aided system we propose in this paper is roughly shown in Figure 3.

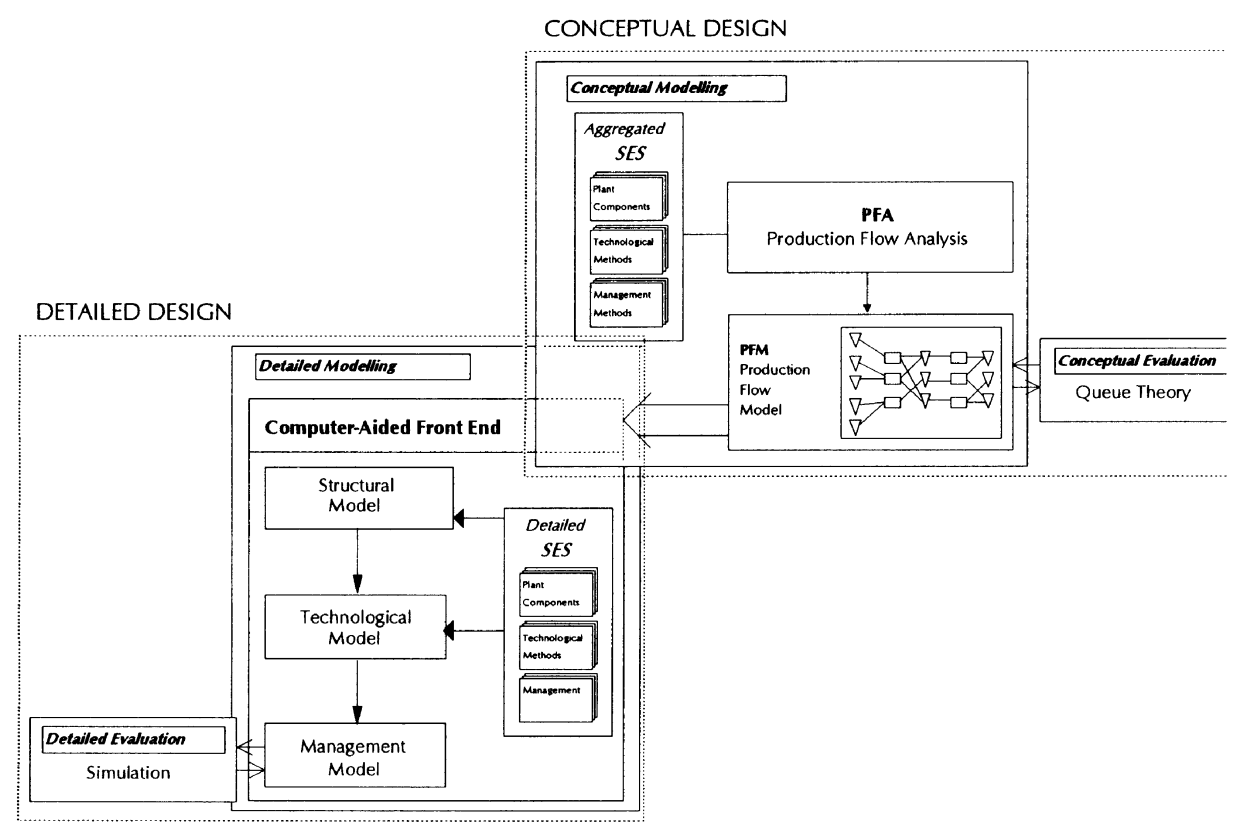

Figure 3 MSEW general architecture.

To meet the need for gradually higher detail levels (Doumeingts et al., 1987), the design process has been divided into two phases:

- a conceptual design phase, including a rough design of the manufacturing system. This phase aims at developing a schematic model of the manufacturing system, called Production Flow Model, based on the information available in an aggregated version of the mfg system SES, and 
- a detailed design phase, including a detailed design of the system which builds on the outcome of the previous phase. In this phase, designers use an extended SES version for manufacturing systems, to develop a detailed system model.

\section{Conceptual design phase}

The conceptual design phase can be further divided into two steps:

- a modelling step and

- an evaluation step.

During the modelling step, a PFA (Production Flow Analysis) module processes general information such as product structure (i.e. a bill of all components making up the end products), gross technological routes of parts and quantitative estimates of the demand for end products to automatically generate a Production Flow Model (PFM), i.e. a simplified model of production flows based on the nature and average capacity of processing agents (operating stations), stocking agents and handling agents. Obviously, the detail level of the resulting PFM module will be determined by the detail level of the input data. The PFA module can also generate a simplified graphic representation of the manufacturing system consisting of a scheme similar to the one shown in Figure 3 (where triangles stand for stocking agents, rectangles for processing agents and lines for handling agents).

When filled in with all the relevant quantitative information, the Production Flow Model can provide indications about the most suitable lay-out for the manufacturing system to be designed, since a visual analysis of the flow type (either criss-crossed or linear) can help the designer choose the general configuration which better meets the requirements of the manufacturing system (process-oriented versus product-oriented lay-out).

The evaluation step must assess the dynamic behaviour of the PFM, for example by performing a functional model analysis based on queue theory, to ensure that the "static" system configuration resulting from the modelling phase is still valid when the system starts moving.

\section{Detailed design phase}

Similarly to the design phase, the detailed design phase can be divided into two further steps:

- a modelling step and

- an evaluation step.

The modelling step requires the biggest creativity effort by the designer.

The quantitative and qualitative information collected during the conceptual design phase turn into constraints to be met and into guidelines for the designer's work. The PFM acts as a reference point in this phase.

The system can only act as an interface spurring the designer's creative process as much as possible. Undoubtedly, a graphical interface is best suited to this purpose. The designer needs to have icons representing the objects of a generic manufacturing system and to create a graphic model of the system to be evaluated. In this case, a bottom-up approach is followed to 
generate the detailed design of the manufacturing system, since the designer has to start from the smallest components making up the system (i.e. the leaves of the SES).

The model resulting from the choice and interconnection of the permanent objects is called a structural model, since it represents the structural - i.e. static - aspect of the manufacturing system. In order to build an exhaustive model from a dynamic viewpoint as well, the structural model has to be supplemented with the technological methods (i.e. procedures which reflect the processing aspect of the part flow and turn a structural model into a technological model) and with management methods (i.e. procedures reflecting the operating aspects of the manufacturing system which merge into the technological model to create a full operating model).

It should be pointed out that it is not reasonable to expect a designer to directly generate a detailed end project starting from the rough outline resulting from the upstream general design phase: several projects with different detail levels will obviously need to be generated, with the most interesting solutions being identified through an iterative trial and error process.

Discrete-event simulation appears to be the most suitable tool for the detailed evaluation step. Starting from the operating model of a given manufacturing system, the code for its simulation model has to be directly and automatically generated in a suitable language. This will enable the designer to rapidly evaluate the "goodness" of the project by using the simulator's performance indicators and to make any changes required for refinement purposes.

\subsection{The MSEW in a concurrent engineering environment}

Unlike traditional design techniques which are sequential, iterative and independent, concurrent engineering (CE) or simultaneous engineering (SE) require a parallel, interactive and cooperative approach to product design. Thus, the $\mathrm{CE}$ approach requires that the product and the manufacturing system life-cycles be coordinated and parallelized due to their strong interactions (Kovacs et al., 1991).

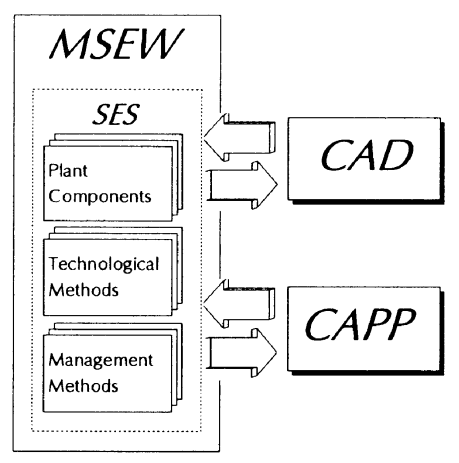

Figure 4 The MSEW in a concurrent-engineering environment. 
Hence the need for communication between the MSEW and the CAD (Computer-Aided Design) and CAPP (Computer-Aided Process Planning) systems used by product designers and process designers (see Figure 4). In fact the design specifications and data provided by these systems could be stored into objects making up the System Entity Structure and later gathered by the MSEW user who will interactively assess their feasibility from the manufacturing system point of view.

\section{PRELIMINARY RESULTS AND CONCLUSIONS}

Some software module of the general architecture described in this paper have been successfully tested in a preliminary and limited implementation of the system:

- a PFA module was developed for the general design phase using an Oracle relational database;

- for a class of automatic assembly lines a SES and a computer-aided front-end device were developed, based on an Gensym Co. G2 shell for expert systems, for the detailed design phase. The automatic generation of the simulation code (based on AT\&T Witness simulation software) was also successfully tested starting from the knowledge base of the expert system.

Prototypes for the remaining elements of the suggested architecture need to be developed; the use of an object-oriented simulation software, providing a better integration with the object-based representation of the manufacturing system, is currently under evaluation and more detailed analyses of the semantics of different manufacturing systems need to be implemented and turned into a structured knowledge base.

In conclusion, the potential benefits offered by the development of a Manufacturing System Engineering Workbench for the computer-aided design of manufacturing systems have been verified to be well grounded and quite evident, thus calling for a stronger research effort to be performed on this subject in the near future.

\section{REFERENCES}

Davenport, T.H. and Short, J.E. (1990) The New Industrial Engineering: Information Technology and Business Process Redesign. Sloan Management Review, 6, 11-27.

Doumeingts, G., Vallespir, B., Darricau, D. and Roboam, M. (1987) Design methodology for Advanced Manufacturing Systems. Computers in Industry, 9, 271-296.

Kovacs, G., Mezgar, I. and Kopsaci, S. (1991) Concurrent Design of Automated Manufacturing Systems using Knowledge Processing Technology. Computers in Industry, $17,205-222$.

McLean, C.R. (1993) Computer-Aided Manufacturing System Engineering, in Advances in Production Management Systems (eds. I.A. Pappas and I.P. Tatsiopoulos), Netherlands: Elsevier Science.

Wang, W., Popplewell, K. and Bell, R. (1993) An integrated multi-view system description approach to approximate factory modelling. International Journal of Computer Integrated Manufacturing, 6(3), 165-174. 
Zeigler, B.P. and Rozenblit, J.W. (1987) Design and Modeling Concepts, in Encyclopedia of Robotics (eds. R. Dorf and S. Nef), John Wiley \& Sons, New York.

Zeigler, B.P.and Zhang, G. (1989) The System Entity Structure: Knowledge Representation for Simulation Modeling and Design, in Artificial Intelligence, Simulation, and Modeling (eds. L.E. Widman, K.A. Loparo and N.R. Nielsen), John Wiley \& Sons, New York.

\section{BIOGRAPHY}

MARCO GARETTI is Professor of Industrial Technology at the Dipartimento di Economia e Produzione of the Politecnico di Milano, Italy. After getting a Mechanical Engineering degree from the Polytechnic in 1971, he began his career within Alfa Romeo, where he dealt with mathematical modelling and the computer-aided design of car bodies. In 1974 he joined the Politecnico di Milano and specialized in production systems design and management, with special emphasis on automated production and CIM. He is a senior member of the Computer and Automated Systems Association of the Society of Mechanical Engineering (CASA/SME), a member of the editorial board of PIXEL (an Italian CAD/CAM magazine) and a member of the International Journal of Production Planning and Control.

$\mathrm{He}$ is actively involved in the organization of seminars and post-graduate courses on production management and automated manufacturing. His current interests include the design of production systems, production management, intelligent manufacturing systems, simulation and CIM.

ANNA BARTOLOTTA is a research fellow of the National Research Council (Consiglio Nazionale delle Ricerche, CNR) at the Dipartimento di Economia e Produzione of the Politecnico di Milano. She received the degree in Management Engineering in 1994 at the Politecnico di Milano presenting a degree thesis entitled "Framework for a Manufacturing System Engineering Workbench". Her research interests include advanced tools and techniques for the design and management of manufacturing systems.

This study was carried out within the framework of a national research program called "Advanced Techniques for the Design and Management of Industrial Production Systems" funded by the Italian Ministry for University and Scientific Research (MURST 40\%). 